\title{
Nanoparticles-Based Naked-Eye Colorimetric Immunoassays for In Vitro Diagnostics
}

\section{Sandeep Kumar Vashist*}

HSG-IMIT - Institut für Mikro- und Informations Technik, Georges-Koehler-Allee 103, 79110 Freiburg, Germany

Immunoassays are the largest class of assays for the detection of protein biomarkers, which play a prominent role in biomedical diagnostics, biosensors and bioanalytical sciences. There has been a rapidly growing interest in naked-eye immunoassays based on the use of nanoparticles (NP) for in vitro diagnostic (IVD) applications [1]. Naked-eye detection, requiring only the human eyes, is foreseen as the critical requirement for the development of next-generation of low-cost IVD kits. The detection of colorimetric product after enzyme-substrate reaction has always been an integral part of conventional Enzymelinked immunosorbent immunoassay (ELISA). But the naked-eye colorimetric detection can only be done reliably at higher analyte concentrations, which makes it unsuitable for ultrasensitive detection. During the last decade, several prospective NP-based strategies have been developed. Although most of these strategies employ gold nanoparticles (AuNPs) [2], there is a continuously evolving trend towards the use of other prospective nanoparticles (such as Ceria $\mathrm{NP}$, graphene, carbon nanotubes) and nanocomposites. NPs form the critical component of naked-eye colorimetric immunoassays as the aggregation of solutions containing low concentrations of NPs displays a clear colour change.

The recent demonstration of plasmonic ELISA for the ultrasensitive detection of disease biomarkers by naked-eye colorimetric detection is a highly prospective development [3]. It is a potential immunoassay format for the developing countries and resource-limited settings as it does not require any sophisticated instruments and is based on the generation of colored solutions of characteristics tonality (Figure 1A). The developed format is based on the linkage of the bio catalytic cycle of enzyme label to the growth of AuNPs in order to obtain blue or redcolored solutions in the presence or absence of the analyte, respectively. The absence of analyte leads to the reduction of Au ions with hydrogen peroxide $\left(\mathrm{H}_{2} \mathrm{O}_{2}\right)$ that results in the formation of non-aggregated AuNPs and the characteristic red color of solution. On the other hand, the presence of analyte leads to the consumption of $\mathrm{H}_{2} \mathrm{O}_{2}$ by catalase, which results in the formation of aggregated AuNPs that is responsible for the blue color of solution (Figure 1B). The developed plasmonic ELISA was demonstrated successfully for the detection of prostate specific antigen (PSA) and HIV-1 capsid antigen p24 in whole serum at ultralow concentration of 1 attogram $/ \mathrm{mL}$. It detected p24 in the sera of HIV-infected patients with much lower limit of detection (LOD) in comparison to the highly-expensive 'gold standard' i.e. nucleic acidbased test. The lower LOD of plasmonic ELISA is due to the higher efficiency of catalase to fine tune the concentration of $\mathrm{H}_{2} \mathrm{O}_{2}$. It can be used for the low-cost ultrasensitive detection of p24 to diagnose HIV infection in the developing countries. The developed technology can also be incorporated in lab-on-a-chip and microfluidic devices, which will lead to the development of fully-integrated immuno-analytical systems for point-of-care (POC) IVD.

Copper $(\mathrm{Cu})$-base click chemistry has also been employed extensively for naked-eye readout of immunoassays [4]. Cu-based click chemistry, where $\mathrm{Cu}$ acts as a catalyst, leads to high sensitive and specific $\mathrm{Cu}$ monoxide $(\mathrm{CuO})$ NP-based immunoassays. The color change in such immunoassays occurs as a result of aggregation of AuNPs in the presence of $\mathrm{Cu}^{\mathrm{II}}$ with sodium ascorbate as the reductant. $\mathrm{Cu}^{\mathrm{II}}$ is released into the solution by $\mathrm{HCl}$ and detected by the detection mixture comprising of azide- and alkyne-functionalized AuNPs and sodium ascorbate. The developed naked-eye immunoassay strategy was tested for the detection of ovalbumin (OVA) and HIV-1 gp41 antigen. The procedure involves the binding of antigen to the 96-well MTP, which is followed sequentially by the binding of primary antibody and $\mathrm{CuO}$ NP-labeled secondary antibody (Figure 2). Thereafter, the CuO NPs were dissolved by the addition of $\mathrm{HCl}$, which leads to the formation of $\mathrm{Cu}^{\mathrm{II}}$ ions that were detected with high sensitivity and specificity by click chemistry. The release of $\mathrm{Cu}^{\mathrm{II}}$ ions leads to the aggregation of AuNPs functionalized with azide and alkyne groups, which changes the color of solution in about $10 \mathrm{~min}$. The developed immunoassay has the same sensitivity and LOD as the absorbance or fluorescence-based MTP readers-based immunoassays. It enables the cost-effective detection of HIV infection without employing any sophisticated instruments.

A personalized food allergen testing platform 'iTube'has also had been demonstrated [5]. It analyses the colorimetric assays performed in test tubes to automatically detect the allergens in food samples using smart phone (Figure 3$)$. It involves a light-weight ( 40 g), compact $(\sim 22 \mathrm{~mm} \times 67 \mathrm{~mm} \times 75 \mathrm{~mm})$ and cost-effective opto-mechanical attachment, which comprises of a low-cost plano-convex lens, two diffused light emitting diodes (LEDs) to vertically illuminate the test and control tubes that are loaded from the side, and circular apertures to spatially control the imaging field-of-view (FOV). The image of the colorimetric solution in the iTube is taken by the smart phone's camera and subsequently processed by a smart application in $<1$ s to determine the allergen concentration in food samples. The iTube platform was employed to determine the concentration of allergens in several commercially-available cookies containing varying amounts (1-25 part per million, ppm) of peanut. It precisely quantified food allergens in less than 20 min with a detection limit of $\sim 1 \mathrm{ppm}$. This promising approach provided a reliable smart phone-based technology for the precise detection of analytes based on colorimetric assays.

The naked-eye detection is ideal for analyte detection in resourcelimited settings. But it has a potential drawback that it is inaccurate for quantifying the exact analyte concentration despite having much lower LOD than conventional ELISA [3]. Therefore, the readout of naked-eye immunoassays has been the bottleneck for developing POC

*Corresponding author: Sandeep Kumar Vashist, HSG-IMIT - Institut für Mikro- und Informationstechnik, Georges-Koehler-Allee 103, 79110 Freiburg, Germany, Tel: 49 761203 7252; Fax: 49761203 73299; E-mail: sandeep.kumar.vashist@hsg-imit.de

Received February 13, 2014; Accepted February 15, 2014; Published February 17,2014

Citation: Sandeep Kumar V (2014) Nanoparticles-Based Naked-Eye Colorimetric Immunoassays for In Vitro Diagnostics. J Nanomed Nanotechnol 5: e133. doi:10.4172/2157-7439.1000e133

Copyright: (c) 2014 Sandeep Kumar V. This is an open-access article distributed under the terms of the Creative Commons Attribution License, which permits unrestricted use, distribution, and reproduction in any medium, provided the original author and source are credited. 
Citation: Sandeep Kumar V (2014) Nanoparticles-Based Naked-Eye Colorimetric Immunoassays for In Vitro Diagnostics. J Nanomed Nanotechnol 5: e133. doi:10.4172/2157-7439.1000e133

(A)

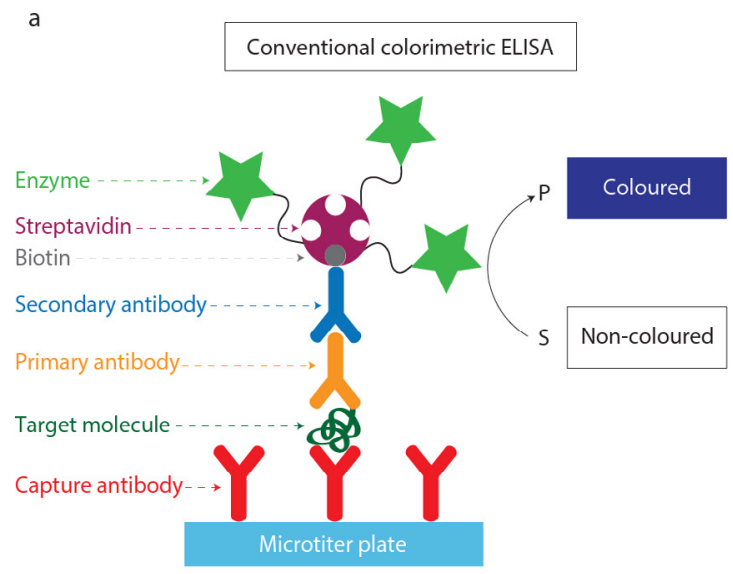

Plasmonic ELISA

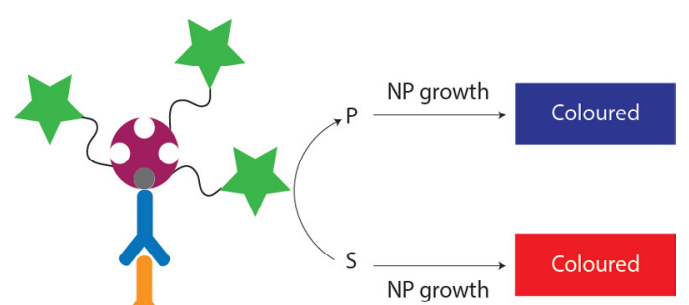

(B)

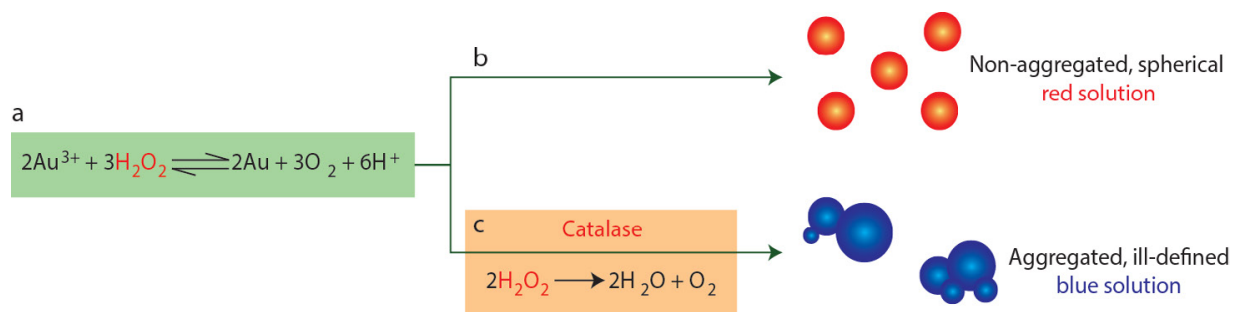

Figure 1: (A) The conventional sandwich ELISA involves the specific binding of target molecules to the capture antibody-bound microtiter plate. This is followed by the detection of bound target molecules by primary antibody followed by the binding of enzyme-bound secondary antibody. The enzyme substrate reaction leads to the formation of colored product. On the other hand, in plasmonic ELISA, the biocatalytic cycle of the enzyme generates colored nanoparticle solutions with characteristic tonality (S, substrate; P, product; NP, nanoparticle). (B) Generation of colored solutions for naked-eye detection. (a) In the presence of hydrogen peroxide, gold ions are reduced. (b) High concentration of hydrogen peroxide favours the formation of non-aggregated, spherical nanoparticles that give rise to a red solution. (c) When the concentration of hydrogen peroxide decreases, for example due to the biocatalytic action of the enzyme catalase, aggregates of nanoparticles are formed and this turns the solution blue. Reproduced with permission from Macmillan Publishers Limited [3].

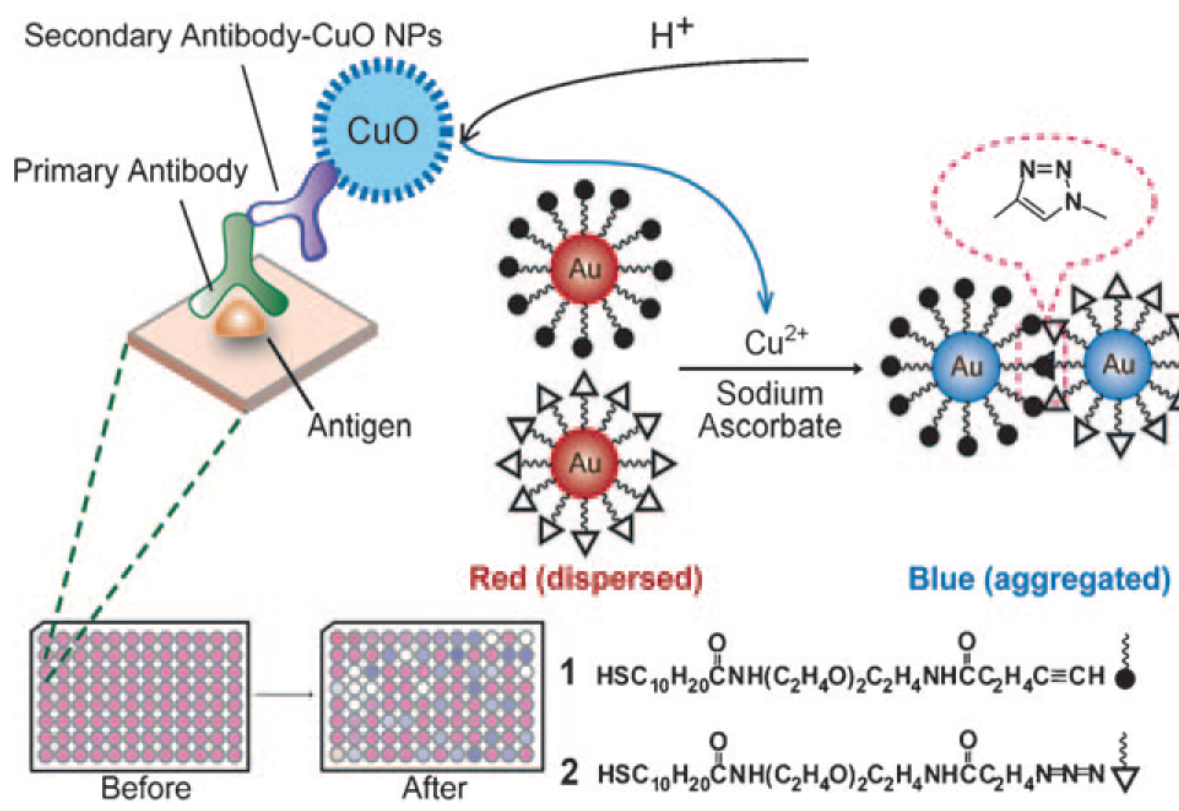

Figure 2: Immunoassay based on CuO-labeled antibody and click chemistry [4]. Reproduced with permission from Wiley-VCH Verlag GmbH \& Co. KGaA, Weinheim. 

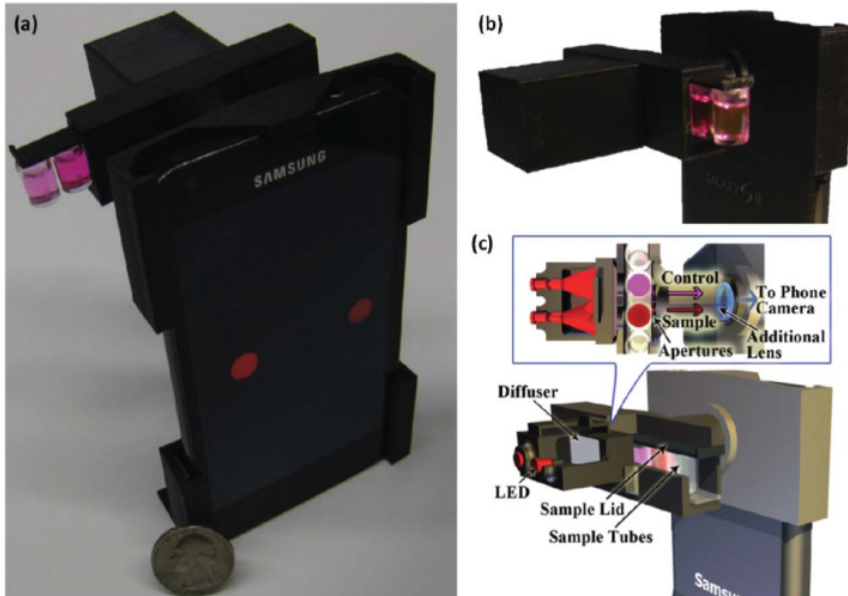

(c)

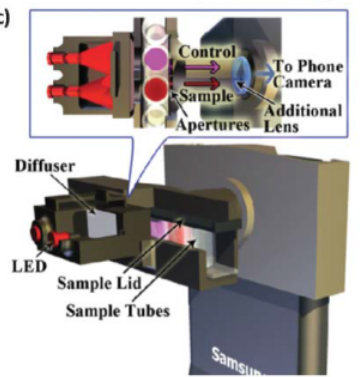

Figure 3: (a) iTube platform, utilizing colorimetric assays and a smart phonebased digital reader [5]. (b) Opti-mechanical attachment $(22 \mathrm{~mm} \times 67 \mathrm{~mm} \times$ $75 \mathrm{~mm}$ ) installed at the back of smart phone. (c) Schematic of iTube platform. Reproduced with permission from The Royal Society of Chemistry.

immunoassays. But this has been solved in the last few years by the arrival of smart systems technologies, such as the use of smart phone camera along with the smart application, which enable highly precise colorimetric detection-based quantification of analyte. However, the NP-based technologies still need to comply with the industrial and healthcare requirements [6]. On the other hand, the last decade has witnessed continuous improvement in immobilization chemistries, immunoassay formats and bioanalytical platforms, which have led to significant advances in the field of immunoassays. These advances have been supplemented by the rapidly evolving technology and business trends towards smart phone-based POC devices for immunoassays [7]. NP-based naked-eye immunoassays will certainly play a pivotal role in the development of cost-effective point-of-care IVD in the near future based on their superior analytical performance, novel characteristic features and plethora of applications. Moreover, the development of recently developed and upcoming supporting technologies will contribute effectively to the commercial success and rapidly growing applications of NPs-based naked-eye immunoassays.

\section{References}

1. TangD, Cui Y, Chen G (2013) Nanoparticle-based immunoassays in the biomedical field. Analyst 138: 981-990.

2. Saha K, Agasti SS, Kim C, Li X, Rotello VM (2012) Gold nanoparticles in chemical and biological sensing. Chem Rev 112: 2739-2779.

3. de la RicaR (2012) Plasmonic ELISA for the ultrasensitive detection of disease biomarkers with the naked eye. Nat Nanotech 7: 821-824.

4. Qu W, Liu Y, Liu D, Wang Z, Jiang X (2011) Copper-mediated amplification allows readout of immunoassays by the naked eye. Angew Chem 123: 3504 3507.

5. Coskun AF, Wong J, Khodadadi D, Nagi R, Tey A, et al. (2013) A personalized food allergen testing on a cellphone. Lab Chip 13: 636-640.

6. Vashist SK, Mudanyali O, Schneider EM, Zengerle R, Ozcan A (2013) Cellphone-based devices for bioanalytical sciences. Anal Bioanal Chem.

7. Vashist SK, Venkatesh AG, Mitsakakis K, Czilwik G, Roth G, et al. (2012) Nanotechnology-based biosensors and diagnostics: technology push versus industrial/healthcare requirements. Bio Nano Sci 2: 115-126. 